\title{
Fitotecnologias: situação atual e perspectivas futuras
}

\author{
Cassiano Augusto Rolim Bernardino ${ }^{a}$, Claudio Fernando Mahlera, Eduardo Ferreira da Silvab, \\ Luís António Balreira Novob,c* \\ a Programa de Pós-Graduação em Engenharia Civil, Instituto Alberto Luiz Coimbra de Pós-Graduação e Pesquisa de Engenharia, \\ Universidade Federal do Rio de Janeiro, Brasil \\ ${ }^{b}$ GeoBioTec, Departamento de Geociências, Universidade de Aveiro, Portugal \\ ${ }^{c}$ Centro de Biotecnologia e Química Fina - Laboratório Associado, Escola Superior de Biotecnologia, Universidade Católica \\ Portuguesa,Porto,Portugal.*novo@ua.pt
}

Recebido: 30 janeiro 2018 / Aceito: 06 maio2018 / Publicado online: 28 maio 2018

\begin{abstract}
Resumo
A degradação do meio ambiente, o aumento da urbanização, a elevada demanda energética, e as alterações climáticas, são atualmente alguns dos principais desafios do ser humano. Além disso, as atividades antropogênicas têm contribuído para a contaminação dos recursos naturais devido a geração, emissão e deposição de poluentes orgânicos e inorgânicos. Nesse sentido, um conjunto de técnicas alicerçadas no uso de plantas (fitotecnologias), têm ganhado grande visibilidade pois oferecem soluções ecológicas e sustentáveis para mitigar esses problemas. Esta revisão sumariza a situação atual dos principais tipos de fitotecnologias, das quais se destacam a fitorremediação, a fitomineração, a utilização da biomassa para produção de energia, e as coberturas verdes. Este trabalho apresenta ainda alguns dos desafios futuros das fitotecnologias, como a sua otimização através de técnicas avançadas de edição genética ou a articulação com as nanotecnologias.
\end{abstract}

Palavras-chave: Fitorremediação, fitomineração, biomassa, telhados verdes, alteração climática.

\section{Phytotechnologies: current status and future challenges}

\begin{abstract}
The degradation of the environment, increased urbanization, global energy demand growth, and climate change, constitute some of the main problems humans are facing today. Furthermore, anthropogenic activities have contributed to the contamination of natural resources due to the generation, emission and deposition of organic and inorganic pollutants. In this sense, a set of technologies based on the use of plants (phytotechnologies), have conquered great visibility because they offer ecological and sustainable solutions to mitigate these problems. This review summarizes the current status of the main types of phytotechnologies, from which phytoremediation, phytomining, biomass for energy generation, and green roofs, stand out. In addition, this work also presents some of the future challenges of phytotechnologies, such as their optimization through advanced gene editing techniques or their combination with nanotechnologies.
\end{abstract}

Keywords: Phytoremediation, phytomining, biomass, green roofs, climate change.

\section{Introdução}

A revolução industrial foi a principal força motriz das alterações climáticas e ambientais antropogênicas, através da mudança das práticas agrícolas e industriais, e do bombeamento de gases de efeito estufa para a atmosfera. $\mathrm{O}$ crescimento da população, resultante da revolução industrial, obrigou à conversão de mais terras para agricultura e desenvolvimento urbano, levando ao desmatamento maciço e à mudança e contaminação do meio ambiente (Pechony e Shindell, 2010; Hooke e Martín-Duque, 2012).

A contaminação do solo tornou-se um grave problema ambiental à escala planetária, devido ao seu impacto negativo sobre os ecossistemas, saúde humana, fertilidade do solo e o equilíbrio socioeconômico. Em todo o mundo, cerca de 52 milhões de hectares, representando mais de $16 \%$ da área total do planeta, são afetados por algum nível de contaminação do solo. Apenas na Europa, há em torno de 2,5 milhões de áreas contaminadas, cujos custos anuais de gestão equivalem a 6,5 bilhões de euros, conforme estimado pela Agência Europeia do Meio Ambiente (European Environment Agency, 2015). As técnicas convencionais para a remediação de solos contaminados (tais como vitrificação, incineração do solo, escavação e transporte para aterro sanitário, entre outras), são frequentemente impraticáveis, financeiramente inviáveis, e ambientalmente danosas (Ali et al., 2013).

$\mathrm{O}$ aumento exponencial da população tem provocado um crescimento das atividades urbanas tais como elevada produção de resíduos sólidos, energia, frota veicular e lixiviados. Consequentemente, essas atividades poluem o ar, água e o solo devido a emissão e a deposição de contaminantes 
orgânicos e inorgânicos. Um dos exemplos para a dimensão desse problema são as alterações climáticas devido as atividades antropogênicas. Uma das causas deste fenômeno negativo provém da emissão de gases como monóxido de carbono, metano, dióxido de carbono e óxidos de nitrogênio para a atmosfera. Isto ocorre devido ao aumento da demanda pela população por produtos agrícolas e industriais. Os principais responsáveis pelas emissões de poluentes na atmosfera são as indústrias de energia, o tráfego de veículos, as atividades agrícolas e mineiras, entre outras fontes, que libertam de forma inadequada compostos e elementos inorgânicos que contaminam o ar, solo e a água. Além disso, contaminantes depositados no solo podem ser transportados, lixiviados ou solubilizados pela água pluvial e assim, poluir os corpos de água superficiais e/ou subterrâneos (Calijuri e Cunha, 2013).

A contaminação do meio ambiente, o agravamento de problemas como a urbanização, demanda energética, e alterações climáticas, promoveram de forma decisiva o estudo e desenvolvimento de um conjunto de estratégias verdes e sustentáveis baseadas no uso de plantas (fitotecnologias), cuja visibilidade e aplicação tem aumentado exponencialmente durante as últimas décadas (Prasad et al., 2010; Koelmel et al., 2015).

\section{Estado da arte das fitotecnologias}

As fitotecnologias são habitualmente associadas à capacidade das plantas para extrair, degradar, imobilizar ou transformar poluentes, pelo que os termos fitotecnologias e fitorremediação são normalmente intercambiáveis. Porém, as aplicações atuais das fitotecnologias transcendem o domínio da fitorremediação, podendo também ser utilizadas para a recuperação de elementos valiosos a partir de rejeitos de minas, solos mineralizados ou contaminados (fitomineração) (Novo et al., 2017), biomassa para geração de energia (Pandey et al., 2016), ou a criação de coberturas verdes para a otimização energética, estrutural e estética de edifícios (Castleton et al., 2010; Figura 1).

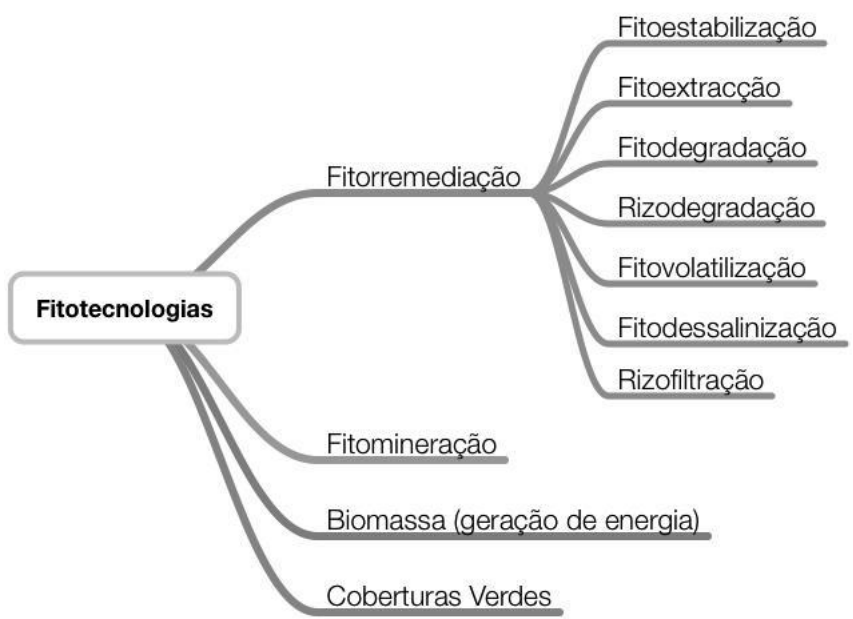

Figura 1. Principais subcategorias das fitotecnologias.

\section{Fitorremediação}

A fitorremediação constitui a base das fitotecnologias, visto que as suas várias categorias oferecem uma vasta gama de opções face a diferentes tipos de contaminantes e condições ambientais. Os principais mecanismos da fitorremediação compreendem: a) fitoestabilização - imobilização dos poluentes na rizosfera pela ação das raízes, bactérias e corretivos do solo; b) fitoextração - extração e acumulação dos contaminantes na parte aérea da planta; c) fitodegradação degradação de xenobióticos através de enzimas no tecido vegetal; d) rizodegradação ou fitoestimulação - degradação de xenobióticos na rizosfera pela ação de microorganismos; e) fitovolatilização - conversão de poluentes para forma volátil e posterior liberação para a atmosfera; f) fitodessalinização remoção de sais em solos salinos através de halófitas; e e) rizofiltração - remoção de contaminantes a partir de ambientes aquáticos poluídos (Ali et al., 2013).

Para utilização destas técnicas é importante definir parâmetros como fatores de bioconcentração (FB) e translocação (FT). O FB e FT são muito úteis para verificar se a espécie de planta em estudo apresenta potencial para imobilizar ou acumular metais (Bernardino et al., 2016). O FB indica a eficiência de uma planta na extração de um metal a partir do solo ou água. Este fator é calculado conforme a equação:

$$
F B=\frac{C_{p}}{C_{s a}}
$$

Onde Cp e Csa são as concentrações de metais na planta $\left(\mathrm{mg} \mathrm{kg}^{-1}\right)$ e no solo ou água $\left(\mathrm{mg} \mathrm{kg}^{-1}\right)$, respectivamente (Peijnenburg e Jager, 2003; Karami et al., 2011).

O FB pode ser utilizado para comparações em casos de crescimento de plantas em solos homogeneizados ou em culturas hidropônicas. Este fator também é uma forma conveniente e confiável de quantificar a diferença relativa na biodisponibilidade dos metais para as plantas (Naseem et al., 2009; van der Ent et al., 2013).

O FT caracteriza a capacidade da planta para transportar os metais da raiz para a parte aérea. Fator de translocação superior a um (FT > 1), indica que a planta é eficiente no transporte dos metais da raiz para a parte aérea (Zhao et al., 2007). Este fator é calculado conforme a equação:

$$
F T=\frac{C_{a}}{C_{r}}
$$

Onde, $\mathrm{Ca}$ e $\mathrm{Cr}$ são concentrações de metais $\left(\mathrm{mg} \mathrm{kg}^{-1}\right)$ na parte aérea e raiz da planta, respectivamente.

Em resumo, FB e FT podem ser usados para estimar o potencial de uma planta para a fitorremediação. Plantas com FB e FT maiores que um (BF e TF $>1$ ), demonstram capacidade para extrair e acumular metais na parte aérea (fitoextração). Por outro lado, plantas com FB maior que um e FT menores que um (FB $>1$ e FT $<1)$, terão potencialidade para imobilizar os contaminantes na raiz.

\section{Fitomineração}

A fitomineração aproveita a capacidade das plantas, particularmente das metalófitas (tolerantes a níveis elevados de metais) e hiperacumuladoras (que apresentam concentrações de um determinado elemento 1-3 ordens de grandeza superiores às encontradas em plantas que crescem no mesmo 
entorno; tabela 1), para recuperar metais valiosos a partir de rejeitos de minas, e solos mineralizados ou contaminados (Novo et al., 2015). Sendo uma tecnologia relativamente recente, a fitomineração explora a capacidade das plantas para acumular metais como ouro, prata, tálio, níquel, entre outros (Tabela 1). A fitomineração de níquel é geralmente considerada a mais viável, devido ao grande número de hiperacumuladoras deste elemento e abundância de solos ultramáficos (ricos em Ni) em todo o mundo (Novo et al., 2017). Neste contexto, uma modalidade da fitomineração, denominada agromineração, tem sido proposta como uma estratégia agrícola que permitiria às comunidades locais "colher" níquel, proporcionando-lhes um benefício econômico (van der Ent et al., 2015).

Tabela 1. Espécies de plantas hiperacumuladoras de diferentes elementos químico.

\begin{tabular}{|c|c|c|c|c|}
\hline Espécie & Elemento & $\begin{array}{c}\text { Concentração* } \\
\left(\mathrm{mg} \mathrm{kg}^{-1}\right)\end{array}$ & Localização & Referência \\
\hline $\begin{array}{l}\text { Potamogeton } \\
\text { oxyphyllus }\end{array}$ & $\mathrm{Ag}$ & 13,5 & Vietnã & Ha et al., 2011 \\
\hline Mentha suavolens & $\mathrm{Ag}$ & 1,9 & Portugal & Pratas et al., 2013 \\
\hline $\begin{array}{l}\text { Cyanoboletus } \\
\text { pulverulentus }\end{array}$ & As & 1300 & $\begin{array}{c}\text { República Tcheca, } \\
\text { França, Portugal e EUA }\end{array}$ & Braeuer et al., 2018 \\
\hline Pteris vittata & As & 3750 & Vietnã & Ha et al., 2011 \\
\hline Trifolium repens & $\mathrm{Au}$ & 27 & Austrália & Piccinin et al., 2007 \\
\hline Coronopus didymus & $\mathrm{Cd}$ & 864 & Índia & Sidhu et al., 2017 \\
\hline Iris ensata & $\mathrm{Cr}$ & 1120 & Korea & Usman et al., 2012 \\
\hline Epilobium hirsutum & $\mathrm{Cu}$ & 1581 & Irã & $\begin{array}{l}\text { Ghaderian e Ghotbi } \\
\text { Ravandi, } 2012\end{array}$ \\
\hline Polypogon fugax & $\mathrm{Cu}$ & 4012 & Irã & $\begin{array}{l}\text { Ghaderian e Ghotbi } \\
\text { Ravandi, } 2012\end{array}$ \\
\hline Pronephrium simplex & $\begin{array}{l}\text { Elementos de terras } \\
\text { raras (Y, La, Ce, Pr, Nd, } \\
\text { Sm, Eu, Gd, Tb, Dy, Ho, } \\
\text { Er, Tm, Yb e Lu) }\end{array}$ & 1200 & China & Lai et al., 2005 \\
\hline Digitalis purpurea & $\mathrm{Fe}$ & 4450 & Portugal & Pratas et al., 2013 \\
\hline Celosia argentea & $\mathrm{Mn}$ & 20228 & China & Liu et al., 2014 \\
\hline $\begin{array}{l}\text { Phytolacca } \\
\text { americana }\end{array}$ & $\mathrm{Mn}$ & 32000 & Estados Unidos & Pollard et al., 2009 \\
\hline Alyssum markgrafii & $\mathrm{Ni}$ & 19100 & $\begin{array}{c}\text { Grécia, Albânia e } \\
\text { Bulgária }\end{array}$ & Bani et al., 2010 \\
\hline Senecio coronatus & $\mathrm{Ni}$ & 12000 & África do Sul & Boyd et al., 2008 \\
\hline Plantago orbignyana & $\mathrm{Pb}$ & 8240 & Peru & Bech et al., 2012 \\
\hline $\begin{array}{l}\text { Potamogeton } \\
\text { oxyphyllus }\end{array}$ & $\mathrm{Pb}$ & 4210 & Vietnã & Ha et al., 2011 \\
\hline $\begin{array}{l}\text { Astragalus } \\
\text { racemosus }\end{array}$ & $\mathrm{Se}$ & 14920 & Estados Unidos & White, 2016 \\
\hline Iberis intermedia & $\mathrm{Ti}$ & 3070 & França & Anderson et al., 1999 \\
\hline Plantago orbignyana & $\mathrm{Zn}$ & 11560 & Peru & Bech et al., 2012 \\
\hline
\end{tabular}

*Concentração máxima na parte aérea da planta para o elemento correspondente.

\section{Biomassa}

O interesse pela agricultura energética aumentou significativamente durante os últimos 20 anos, devido ao seu potencial uso como fonte de energia renovável limpa e ecológica. Devido à crescente preocupação com a demanda e segurança energética, aquecimento global e efeitos associados às mudanças climáticas; a energia renovável baseada em biomassa tem integrado a agenda científica internacional, de modo a enfrentar esses desafios (Pandey et al., 2016; Tripathi et al., 2016). Além disso, entre os vários tipos de energia renovável, a energia baseada em biomassa é uma das energias mais importantes devido à profusão de recursos. $\mathrm{O}$ aumento da demanda de energia nos setores doméstico, industrial, transporte e agrícola, exigiu também a rápida implementação de opções bioenergéticas.
Globalmente, espera-se que a energia renovável à base de biomassa se expanda dos 50 EJ ano-1 atuais para 80-160 EJ ano-1 em 2050 (Pandey et al., 2016). Segundo o Instituto Brasileiro de Petróleo, Gás e Biocombustíveis, a biomassa em termos de produção de energia primária no Brasil em 2015 foi em torno de 74 x 106 tep, correspondendo a $26 \%$ da geração na matriz energética no país (Ibp, 2015). Destaca-se também a produção de etanol na safra 2016/2017, que foi de 27,81 bilhões de litros. Este biocombustível proveniente da cana de açúcar tem um papel importante na matriz energética Brasileira, pois pode ser utilizado como combustível nos veículos flex-fuel (hidratado), misturado com a gasolina, com o objetivo de baratear o combustível, aumentar sua octanagem e reduzir a emissão de poluentes (anidro), além da utilização na fabricação de tintas, vernizes, solventes, e outros produtos 
(CONAB, 2017).

A biomassa pode ser obtida através de vegetais lenhosos e não lenhosos, como madeira e resíduos relacionados, assim como a partir de resíduos orgânicos de origem agrícola, industrial e urbana, incluindo a utilização de plantas pósfitorremediação. Os biofluidos, como os óleos vegetais obtidos a partir da mamona, soja, constituem fontes alternativas de biomassa (Cortez et al., 2008).

A conversão de biomassa em energia é realizada utilizando três tecnologias de processo principais: termoquímica, bioquímica/biológica e extração mecânica. Os três processos fundamentais para a conversão termoquímica são combustão, pirólise e gaseificação. A combustão é a queima de biomassa na presença de oxigênio, e é usada para converter a energia química armazenada em biomassa em energia térmica, energia mecânica e também em eletricidade por diferentes processos e dispositivos, como por exemplo, fornos, fogões, turbinas a vapor, e caldeiras (Kumar et al., 2015). O processo de pirólise consiste na decomposição térmica de componentes orgânicos da biomassa em uma faixa de temperatura que varia de $300^{\circ} \mathrm{C}$ até $1000^{\circ} \mathrm{C}$, em atmosfera inerte, e tem por objetivo gerar bioprodudos (bio-óleo, biocarvão e bio-gás) com maior valor agregado.

O bio-óleo pode ser usado em motores, turbinas e como matéria-prima para refinarias. O biocarvão pode ser utilizado como combustível sólido, fertilizante orgânico e bioadsorvente. O bio-gás pode ser empregado para suprir as necessidades no aquecimento do reator de pirólise (Bahng et al., 2009; Bernardino et al., 2017). A gaseificação é a conversão de biomassa em uma mistura de gás combustível, pela oxidação parcial da biomassa a altas temperaturas, na faixa de $800-900{ }^{\circ} \mathrm{C}$, que tem por objetivo produzir maior quantidade de gás. $\mathrm{O}$ gás produzido pode ser queimado diretamente ou usado como combustível para motores e turbinas. Além disso, pode ser usado como gás de síntese para a produção de produtos químicos como o metanol. Outro fator importante é a utilização da gaseificação em combinação com a combustão com o propósito de se alcançar maior eficiência na produção de energia (Kumar et al., 2015; Watson et al., 2018).

Em relação a conversão bioquímica/biológica, destaca-se a fermentação e a digestão anaeróbica. A fermentação é utilizada para produzir etanol a partir de culturas de açúcar (cana-de-açúcar) e culturas de amido (milho), utilizando leveduras (como por exemplo, Saccharromyces cerevisiae). A digestão anaeróbica, ocorre em meio inerte, transformando o material orgânico em biogás (metano), através de bactérias. O biogás pode ser usado diretamente no motor a gás de ignição e turbinas (Kumar et al., 2015).

A extração é um processo de conversão mecânica, no qual, o óleo é obtido a partir de sementes de várias biomassas como soja, girassol, mamona, milho, pinhão-manso, caroço de algodão, a canola, babaçu, buriti, dendê, macaúba, e amendoim, entre outras. O processo de transesterificação alcalina destes óleos vegetais gera o biodiesel, que pode ser classificado como qualquer combustível alternativo, de natureza renovável, que possa oferecer vantagens socioambientais ao ser empregado na substituição total ou parcial do diesel de petróleo em motores do ciclo Diesel. No Brasil, a Agência Nacional de Petróleo, Gás Natural e Biocombustível (ANP) regulamentou a utilização de biodiesel em mistura com diesel de petróleo, autorizando o seu uso em misturas como o objetivo de torná-lo obrigatório a partir de 2008. A produção anual de biodiesel no Brasil gira em torno de 4,1 bilhões de litros. A mistura de biodiesel tem gerado impactos ambientais positivo como a redução das emissões de gases de efeito estufa (Kumar et al., 2015; Ramos et al., 2017).

\section{Coberturas verdes}

Devido ao rápido crescimento econômico, o planeta tem sofrido uma pronunciada urbanização que vai reduzindo as áreas verdes. A escassez de vegetação nas áreas urbanas causa uma diminuição na captação da chuva pela copa das árvores e transpiração, resultando no aumento da temperatura e redução da umidade do ar. Além disso, $40 \%$ do consumo energético mundial está associado à construção e manutenção de edifícios, sendo estes responsáveis por $33 \%$ das emissões globais de gases estufa (Castleton et al., 2010; Berardi et al., 2014). Estes problemas podem ser parcialmente resolvidos através da introdução de plantas e solo nas superfícies dos telhados, alterando as suas propriedades e aumentando a sustentabilidade dos edifícios. As coberturas verdes (ou telhados verdes), são geralmente compostas por diferentes camadas que incluem vegetação, substrato, tecido filtrante, material de drenagem, barreira radicular e isolamento.

As coberturas verdes melhoram o desempenho térmico dos edifícios, permitem o aproveitamento das águas pluviais (incluindo a filtragem de contaminantes), diminuem o ruído, e atenuam a poluição atmosférica, entre outras vantagens (Berardi et al., 2014; Vijayaraghavan, 2016). A tabela 2 sumariza as principais vantagens das coberturas verdes.

\section{Perspectivas futuras}

O futuro das fitotecnologias passa essencialmente por uma mudança conceitual, que integre de forma clara e indissociável a sua vertente econômica. Apesar dos avanços científicos alcançados durante as últimas décadas, da sua adequabilidade e atualidade no âmbito dos problemas mais prementes do século 21 , e da eficiência e rentabilidade demonstrada em inúmeros casos de estudo, as fitotecnologias necessitam se tornar uma ferramenta global, cujo apelo econômico iguale o ecológico. Esse desafio exige maior interação entre a academia, indústria, investidores, sociedade, e instituições governamentais competentes.

A curto prazo, diferentes processos das fitotecnologias podem se beneficiar de técnicas avançadas de edição genética (ex. CRISPR), capazes de melhorar características como a produção de biomassa, hiperacumulação, ou tolerância aos contaminantes. A utilização de culturas energéticas para processos de fitorremediação, a articulação das fito com as nanotecnologias, o incremento da biofortificação (melhoria do valor nutricional das plantas através da sua modificação artificial ou genética) como uma mais-valia agrícola, e a estandardização das coberturas verdes como uma componente obrigatória das edificações em meio urbano, podem também contribuir significativamente para o desenvolvimento das fitotecnologias e a geração de novas oportunidades. 
Tabela 2. Benefícios das coberturas verdes

\begin{tabular}{|c|c|c|}
\hline Benefício & Descrição & Referência \\
\hline $\begin{array}{l}\text { Redução das } \\
\text { emissões de } \\
\text { carbono }\end{array}$ & $\begin{array}{l}\text { As coberturas verdes promovem a redução das emissões de carbono devido ao menor } \\
\text { consumo de energia e ao processo de fotossíntese que usa dióxido de carbono } \\
\text { atmosférico. }\end{array}$ & $\begin{array}{l}\text { Besir e Cuce, } \\
\quad 2018\end{array}$ \\
\hline $\begin{array}{l}\text { Atenuação das } \\
\text { águas pluviais }\end{array}$ & $\begin{array}{l}\text { As coberturas verdes retêm a água da chuva, e limitam o fluxo máximo, reduzindo } \\
\text { assim o risco de inundações. Além disso, as plantas desempenham um papel } \\
\text { significativo na redução do escoamento em função da capacidade de interceptação e } \\
\text { retenção de água. }\end{array}$ & $\begin{array}{l}\text { Vijayaraghavan, } \\
2016 b\end{array}$ \\
\hline $\begin{array}{l}\text { Diminuição do } \\
\text { consumo } \\
\text { energético }\end{array}$ & $\begin{array}{l}\text { Devido ao sombreamento, melhor isolamento e maior massa térmica, causados pela } \\
\text { presença da camada de vegetação, a penetração da radiação solar é controlada, e } \\
\text { consequentemente o consumo energético do edifício é otimizado. }\end{array}$ & $\begin{array}{l}\text { Berardi et al., } \\
\quad 2014 \mathrm{~b}\end{array}$ \\
\hline $\begin{array}{l}\text { Captura de } \\
\text { poeiras }\end{array}$ & $\begin{array}{l}\text { As coberturas verdes podem reduzir a concentração atmosférica de poeiras através da } \\
\text { sua captura. A área e densidade da cobertura verde, assim como as características das } \\
\text { plantas selecionadas determinam a eficiência no sequestro das poeiras. }\end{array}$ & Xiao et al., 2014 \\
\hline $\begin{array}{l}\text { Redução da } \\
\text { poluição }\end{array}$ & $\begin{array}{l}\text { As coberturas verdes atuam como uma barreira de retenção para o nitrogênio e metais } \\
\text { como cádmio, cobre, zinco e chumbo, entre outros. Reduzem também os efeitos da } \\
\text { chuva ácida, elevando o valor do } \mathrm{pH} \text { de } 5 \text { a } 6 \text { na água da chuva, para } 7 \text { a } 8 \text { na água de } \\
\text { escoamento. }\end{array}$ & $\begin{array}{l}\text { Li e Yeung, } \\
\text { 2014; } \\
\text { Vijayaraghavan, } \\
\text { 2016b }\end{array}$ \\
\hline $\begin{array}{l}\text { Isolamento } \\
\text { acústico }\end{array}$ & $\begin{array}{l}\text { Reduzem a poluição sonora em espaços urbanos decorrentes do tráfego rodoviário, } \\
\text { ferroviário e aéreo, devido a absorção de ondas sonoras nas suas diferentes camadas }\end{array}$ & $\begin{array}{l}\text { Vijayaraghavan, } \\
2016 b\end{array}$ \\
\hline $\begin{array}{l}\text { Preservação } \\
\text { Ecológica }\end{array}$ & $\begin{array}{l}\text { Estudos relatam os benefícios das coberturas verdes quanto a melhoria da qualidade } \\
\text { ambiental e preservação ecológica em áreas urbanas. Há um aumento da biodiversidade } \\
\text { e redução das perdas de habitat. Recentemente, estão também associadas ao aumento } \\
\text { da agricultura urbana }\end{array}$ & $\begin{array}{l}\text { Berardi et al., } \\
\quad 2014 \mathrm{~b}\end{array}$ \\
\hline
\end{tabular}

\section{Agradecimentos}

Os autores agradecem o apoio do programa FEDER/Interreg Sudoe através do projeto PhytoSUDOESOE1/P5/E0189; da Fundação para a Ciência e Tecnologia (FCT), através da bolsa $\mathrm{n}^{\circ}$ SFRH/BPD/103476/2014; e do Conselho Nacional de Desenvolvimento Científico e Tecnológico (CNPq), através da bolsa $n^{\circ}$ 141368/2014-4.

\section{Referências}

Ali, H.; Khan, E.; Sajad, M.A. 2013. Phytoremediation of heavy metalsConcepts and applications. Chemosphere, 91(7): 869-881.

Anderson, C.W.; Brooks, R.; Chiarucci, a; LaCoste, C.; Leblanc, M.; Robinson, B..; Stewart, R. 1999. Phytomining for nickel, thallium and gold. Journal of Geochemical Exploration, 67(1-3): 407-415.

Bahng, M.K.; Mukarakate, C.; Robichaud, D.J.; Nimlos, M.R. 2009. Current technologies for analysis of biomass thermochemical processing: a review. Analytica Chimica Acta, 651: 117-138.

Bani, A.; Pavlova, D.; Echevarria, G.; Mullaj, A.; Reeves, R.D.; Morel, J.L.; Sulçe, S. 2010. Nickel hyperaccumulation by the species of Alyssum and Thlaspi (Brassicaceae) from the ultramafic soils of the Balkans. Botanica Serbica, 34(1): 3-14.

Bech, J.; Duran, P.; Roca, N.; Poma, W.; Sánchez, I.; Barceló, J.; Poschenrieder, C. 2012. Shoot accumulation of several trace elements in native plant species from contaminated soils in the Peruvian Andes. Journal of Geochemical Exploration, 113: 106-111.

Berardi, U.; GhaffarianHoseini, A.; GhaffarianHoseini, A. 2014. State-ofthe-art analysis of the environmental benefits of green roofs. Applied Energy, 115: 411-428.

Bernardino, C.A.R.; Mahler, C.F.; Preussler, K.H.; Novo, L.A.B. 2016. State of the Art of Phytoremediation in Brazil. Review and Perspectives. Water, Air, \& Soil Pollution, 227: 272.

Bernardino, C.A.R.; Mahler, C.F.; Veloso, M.C.C.; Romeiro, G.A. 2017. Preparation of Biochar from Sugarcane By-product Filter Mud by Slow Pyrolysis and Its Use Like Adsorbent. Waste and Biomass Valorization, 8(7): 2511-2521.

Besir, A.B.; Cuce, E. 2018. Green roofs and facades: A comprehensive review. Renewable and Sustainable Energy Reviews, 82: 915-939.

Boyd, R.S.; Davis, M.A.; Balkwill, K. 2008. Elemental patterns in Ni hyperaccumulating and non-hyperaccumulating ultramafic soil populations of Senecio coronatus. South African Journal of Botany, 74(1): $158-162$.

Braeuer, S.; Goessler, W.; Kameník, J.; Konvalinková, T.; Žigová, A.; Borovička, J. 2018. Arsenic hyperaccumulation and speciation in the edible ink stain bolete (Cyanoboletus pulverulentus). Food Chemistry, 242(June 2017): 225-231.

Calijuri, M. do C.; Cunha, D.G.F. 2013. Engenharia Ambiental: conceitos, tecnologia e gestão. Rio de Janeiro: Elsevier.

Castleton, H.F.; Stovin, V.; Beck, S.B.M.; Davison, J.B. 2010. Green roofs; building energy savings and the potential for retrofit. Energy and Buildings, 42(10): 1582-1591.

CONAB. 2017. Acompanhamento da Safra Brasileira da Cana-de- açúcar Safra 2017/2018. Companhia Nacional de Abastecimento (Vol. 4).

Cortez, L.A.B.; Lora, E.E.S.; Gómez, E.O. 2008. Biomassa para energia. São Paulo: Unicamp.

European Environment Agency. 2015. Progress in management of contaminated sites. European Environment Agency.

Ghaderian, S.M.; Ghotbi Ravandi, A.A. 2012. Accumulation of copper and other heavy metals by plants growing on Sarcheshmeh copper mining area, Iran. Journal of Geochemical Exploration, 123: 25-32.

Ha, N.T.H.; Sakakibara, M.; Sano, S.; Nhuan, M.T. 2011. Uptake of metals and metalloids by plants growing in a lead-zinc mine area, Northern Vietnam. Journal of Hazardous Materials, 186(2-3): 1384-1391.

Hooke, R.L.; Martín-Duque, J.F. 2012. Land transformation by humans: A review. GSA Today, 12(12): 4-10.

Ibp. 2015. Evolução da produção de energia primária no Brasil.

Karami, N.; Clemente, R.; Moreno-Jiménez, E.; Lepp, N.W.; Beesley, L. 2011. Efficiency of green waste compost and biochar soil amendments for reducing lead and copper mobility and uptake to ryegrass. Journal of Hazardous Materials, 191: 41-48.

Koelmel, J.; Prasad, M.N. V.; Pershell, K. 2015. Bibliometric Analysis of Phytotechnologies for Remediation: Global Scenario of Research and Applications. International Journal of Phytoremediation, 17(2): 145-153.

Kumar, A.; Kumar, N.; Baredar, P.; Shukla, A. 2015. A review on biomass 
energy resources, potential, conversion and policy in India. Renewable and Sustainable Energy Reviews, 45: 530-539.

Lai, Y.; Wang, Q.; Yan, W.; Yang, L.; Huang, B. 2005. Preliminary study of the enrichment and fractionation of REEs in a newly discovered REE hyperaccumulator Pronephrium simplex by SEC-ICP-MS and MALDITOF/ESI-MS. Journal of Analytical Atomic Spectrometry, 20(8): 751753.

Li, W.C.; Yeung, K.K.A. 2014. A comprehensive study of green roof performance from environmental perspective. International Journal of Sustainable Built Environment, 3: 127-134.

Liu, J.; Shang, W.; Zhang, X.; Zhu, Y.; Yu, K. 2014. Mn accumulation and tolerance in Celosia argentea Linn.: A new Mn-hyperaccumulating plant species. Journal of Hazardous Materials, 267: 136-141.

Naseem, S.; Bashir, E.; Shireen, K.; Shafiq, S. 2009. Soil-plant relationship of Pteropyrum olivieri, a serpentine flora of Wadh, Balochistan, Pakistan and its use in mineral prospecting. Studia Universitatis Babes-Bolyai, Geologia, 54(2): 33-39.

Novo, L.A.B.; Castro, P.M.L.; Alvarenga, P.; da Silva, E.F. 2017. Phytomining of Rare and Valuable Metals. In A. A. Ansari, S. S. Gill, R. Gill, G. R. Lanza, \& L. Newman (Eds.), Phytoremediation Management of Environmental Contaminants, 5: 469-486. Cham: Springer International Publishing.

Novo, L.A.B.; Mahler, C.F.; González, L. 2015. Plants to harvest rhenium: scientific and economic viability. Environmental Chemistry Letters, 13(4): 439-445. DOI: https://doi.org/10.1007/s10311-015-0517-3

Pandey, V.C.; Bajpai, O.; Singh, N. 2016. Energy crops in sustainable phytoremediation. Renewable and Sustainable Energy Reviews, 54: 5873.

Pechony, O.; Shindell, D.T. 2010. Driving forces of global wildfires over the past millennium and the forthcoming century. Proceedings of the National Academy of Sciences, 107(45): 19167-19170.

Peijnenburg, W.J.G.M.; Jager, T. 2003. Monitoring approaches to assess bioaccessibility and bioavailability of metals: Matrix issues. Ecotoxicology and Environmental Safety, 56: 63-77.

Piccinin, R.C.R.; Ebbs, S.D.; Reichman, S.M.; Kolev, S.D.; Woodrow, I.E.; Baker, A.J.M. 2007. A screen of some native Australian flora and exotic agricultural species for their potential application in cyanide-induced phytoextraction of gold. Minerals Engineering, 20(14): 1327-1330.

Pollard, A.J.; Stewart, H.L.; Roberson, C.B. 2009. Manganese Hyperaccumulation in Phytolacca americana L. from the Southeastern United States. Northeastern Naturalist, 16(5): 155-162.

Prasad, M.N.V.; Freitas, H.; Fraenzle, S.; Wuenschmann, S.; Markert, B. 2010. Knowledge explosion in phytotechnologies for environmental solutions. Environmental Pollution, 158(1): 18-23.

Pratas, J.; Favas, P.J.C.; D’Souza, R.; Varun, M.; Paul, M.S. 2013. Phytoremedial assessment of flora tolerant to heavy metals in the contaminated soils of an abandoned $\mathrm{Pb}$ mine in Central Portugal. Chemosphere, 90(8): 2216-2225. https://doi.org/10.1016/j.chemosphere.2012.09.079

Ramos, L.P.; Kothe, V.; César-Oliveira, M.A.F.; Muniz-Wypych, A.S.; Nakagaki, S.; Krieger, N.; Cordeiro, C.S. 2017. Biodiesel: Raw Materials, Production Technologies and Fuel Properties. Revista Virtual de Química, 9(1): 317-369.

Rezania, S.; Taib, S.M.; Md Din, M.F.; Dahalan, F.A.; Kamyab, H. 2016. Comprehensive review on phytotechnology: Heavy metals removal by diverse aquatic plants species from wastewater. Journal of Hazardous Materials, 318: 587-599.

Sidhu, G.P.S.; Singh, H.P.; Batish, D.R.; Kohli, R.K. 2017. Tolerance and hyperaccumulation of cadmium by a wild, unpalatable herb Coronopus didymus (L.) Sm. (Brassicaceae). Ecotoxicology and Environmental Safety, 135: 209-215.

Tripathi, V.; Edrisi, S.A.; Abhilash, P.C. 2016. Towards the coupling of phytoremediation with bioenergy production. Renewable and Sustainable Energy Reviews, 57: 1386-1389.

Usman, A.R.A.; Lee, S.S.; Awad, Y.M.; Lim, K.J.; Yang, J.E.; Ok, Y.S. 2012. Soil pollution assessment and identification of hyperaccumulating plants in chromated copper arsenate (CCA) contaminated sites, Korea. Chemosphere, 87(8): 872-878.

Van der Ent, A.; Baker, A.J.M.; Reeves, R.D.; Chaney, R.L.; Anderson, C.W.N.; Meech, J.A.; Mulligan, D.R. 2015. Agromining: Farming for
Metals in the Future? Environmental Science \& Technology, 49(8): 47734780 .

Van der Ent, A.; Baker, A.J.M.; Reeves, R.D.; Pollard, a. J.; Schat, H. 2013. Hyperaccumulators of metal and metalloid trace elements: Facts and fiction. Plant and Soil, 362(1-2): 319-334.

Vijayaraghavan, K. 2016. Green roofs: A critical review on the role of components, benefits, limitations and trends. Renewable and Sustainable Energy Reviews, 57: 740-752.

Watson, J.; Zhang, Y.; Si, B.; Chen, W.T.; de Souza, R. 2018. Gasification of biowaste: A critical review and outlooks. Renewable and Sustainable Energy Reviews, 83: 1-17.

White, P.J. 2016. Selenium accumulation by plants. Annals of Botany, 117(2): 217-235.

Xiao, M.; Lin, Y.; Han, J.; Zhang, G. 2014. A review of green roof research and development in China. Renewable and Sustainable Energy Reviews, 40: 633-648.

Zhao, G.Q.; Ma, B.L.; Ren, C.Z. 2007. Growth, gas exchange, chlorophyll fluorescence, and ion content of naked oat in response to salinity. Crop Science, 47(1): 123-131. 